\title{
Effective medium theory for elastic metamaterials in two dimensions
}

\author{
Ying Wu, Yun Lai, and Zhao-Qing Zhang* \\ Department of Physics, Hong Kong University of Science and Technology, Clear Water Bay, Kowloon, Hong Kong, China
}

(Received 21 March 2007; revised manuscript received 9 August 2007; published 13 November 2007)

\begin{abstract}
An effective medium theory is developed which goes beyond the quasistatic limit to accurately predict the unusual properties of certain elastic metamaterials in two dimensions. The theory's validity is numerically verified through band-structure calculations for three different elastic metamaterials. The theory shows that the effective bulk modulus $\kappa_{e}$, shear modulus $\mu_{e}$, and mass density $\rho_{e}$ can be made negative near resonances by choosing appropriate resonant scatterers, leading to eight possible types of wave propagation. The theory not only provides a convenient tool to search for new metamaterials with desired properties, it also gives a unified physical picture of these properties. Two examples are presented: one possesses large band gaps at low frequencies; the other exhibits two regions of negative refraction, i.e., one for both longitudinal and transverse waves and the other for longitudinal waves only.
\end{abstract}

DOI: 10.1103/PhysRevB.76.205313

PACS number(s): 43.35.+d

\section{INTRODUCTION}

During the past decade, our understanding of the propagation of classical waves in complex media has seen enormous development. Recently, artificial subwavelength electromagnetic (EM) materials, denoted metamaterials, have significantly broadened the range of material responses found in nature. ${ }^{1,2}$ Through the use of different metamaterials, various novel concepts have been successfully demonstrated, including left-handed (LH) materials which exhibit simultaneous negative permittivity and permeability. ${ }^{1,2}$

LH materials can support unusual phenomena like negative refraction ${ }^{1}$ and subwavelength focusing. ${ }^{2}$ The working principle behind these unusual phenomena is the concept of homogenization. ${ }^{3}$ It has been successfully demonstrated that a LH metamaterial behaves like a homogeneous medium capable of producing negative effective permittivity $\varepsilon_{\text {eff }}$ and effective permeability $\mu_{\text {eff }}$ simultaneously in a frequency range near some resonances of the building blocks of the metamaterial. ${ }^{4}$ The building block of a metamaterial normally consists of one or more scatterers together with a background medium forming a unit cell. The first LH metamaterial was fabricated by using a split ring resonator and a thin conducting wire as the unit cell. ${ }^{1}$

Knowing the effective medium parameters of a metamaterial is useful for studying its wave propagation characteristics. For example, in the study of superlensing, it is simple and convenient to describe a slab of metamaterial in terms of an effective medium and use its $\varepsilon_{\text {eff }}$ and $\mu_{\text {eff }}$ in Maxwell equations. $^{2}$ Since the unusual properties of a metamaterial arise from the built-in resonances of its building blocks, those effective medium theories which are derived in the quasistatic limit are no longer suitable. In the quasistatic limit, the wavelengths in both the scatterers and the background are much larger than the size of the unit cell itself. However, this is no longer true for metamaterials at frequencies near the resonances of the building block. Thus, an effective medium theory for a metamaterial should go beyond the quasistatic limit. To date, various methods have been proposed for studying the effective medium properties of EM metamaterials. ${ }^{5-8}$ Recently, the acoustic analog of metamate- rials and the corresponding effective medium theories have also been explored. ${ }^{9,10}$ An acoustic metamaterial made of silicone rubber spheres suspended in water has been demonstrated to possess negative effective mass density and negative effective modulus simultaneously. ${ }^{9}$

Compared with the work on EM metamaterials, much less study has been devoted to elastic metamaterials. Here, the term "elastic metamaterials" means that the host matrix of the building block is a solid material. The solid host makes the whole system elastic for small stresses. This results in three independent effective parameters: effective bulk modulus $\kappa_{e}$, effective shear modulus $\mu_{e}$, and effective mass density $\rho_{e}$. This is different from the acoustic metamaterials mentioned earlier, where the host medium is a fluid and the system can be described by two effective parameters, the effective bulk modulus and effective mass density. ${ }^{9,10}$ In our definition of elastic and acoustic metamaterials, the scatterers (or inclusions) that are responsible for producing built-in resonances are isolated in the host medium. Our definitions do not include the case when the scatterers (or inclusions) are not isolated and become indistinguishable from the host.

The three independent effective parameters in elastic metamaterials can give rise to richer physics than in their EM and acoustic counterparts. The negative values of each single effective parameter and their various combinations can produce eight types of wave propagation, as will be discussed later. However, to date, only a few kinds of elastic metamaterials have been explored. A kind of locally resonant sonic material which can support large band gaps at low frequencies has been reported. ${ }^{11,12}$ The building block of such an elastic metamaterial consists of a rubber-coated lead sphere embedded in an epoxy host. An analytical model based on a special configuration has been proposed to show that the effective mass density of such a metamaterial can turn negative near the resonances of the building block. ${ }^{13}$ However, since both the sphere and the matrix were considered as almost rigid, the proposed model cannot predict the other two effective parameters, and may not apply to other kinds of elastic metamaterials. In order to design a new metamaterial with certain desired propagation properties, one needs to know how to engineer the resonant properties of the building blocks to produce the desired effective parameters. 
This would be facilitated by a valid effective medium theory which relates all three effective medium parameters to the resonant properties of the building block. However, most of the existing effective medium theories for elastic waves were derived in the quasistatic limit, ${ }^{14-18}$ and, therefore, are not suitable for describing an elastic metamaterial.

In this work, an effective medium theory for certain twodimensional elastic metamaterials was developed. The system consists of cylindrical scatterers arranged in a triangular lattice embedded in a solid host. The elastic parameters of both the scatterers and the host were assumed isotropic. The theory relates the effective medium parameters, $\kappa_{e}, \mu_{e}$, and $\rho_{e}$, to the scattering properties of the scatterers directly. In order to check the validity of the theory, band-structure calculations for three different metamaterials were performed. The results show that the theory is capable of accurately reproducing the band-structure results even at frequencies close to the built-in resonances. Further, the theory was shown to provide useful guidance in searching for metamaterials with desired properties by altering the scattering properties of the scatterers. This will be demonstrated through the design of two kinds of metamaterials: one possessing large band gaps at low frequencies and the other exhibiting two regions of negative refraction.

The derivation of the effective medium theory is presented in Sec. II. The application of the theory to a simple metamaterial is given in Sec. III. Section IV considers two more complicated metamaterials, in which simple scatterers are replaced by coated scatterers. Finally, the conclusions are summarized in Sec. V.

\section{EFFECTIVE MEDIUM THEORY}

The two-dimensional elastic metamaterial considered in this study is composed of cylindrical inclusions of radius $r_{s}$ with $\left(\rho_{s}, \mu_{s}, \kappa_{s}\right)$ arranged in a triangular lattice in the $x-y$ plane embedded in a homogeneous matrix of $\left(\rho_{0}, \mu_{0}, \kappa_{0}\right)$. Here, $\rho$ denotes mass density, $\mu$ represents shear modulus, and $\kappa$ denotes bulk modulus satisfying $\kappa=\lambda+\mu$, where $\lambda$ represents the Lamé constant. Due to the translational symmetry along the cylinder's axis, say, $z$ axis, the elastic modes in the system can be decoupled into a scalar part, $z$ mode, and a vector part, $x y$ mode, with vibrations along the $z$ axis and in the $x-y$ plane, respectively. Here, the more complicated case of the $x y$ mode is studied. ${ }^{19,20}$ The displacement in $x-y$ plane can be described by the following wave equation:

$$
\begin{aligned}
\rho(\vec{r}) \frac{\partial^{2} u_{i}(\vec{r})}{\partial t^{2}}= & \nabla \cdot\left[\mu(\vec{r}) \nabla u_{i}(\vec{r})\right]+\nabla \cdot\left(\mu(\vec{r}) \frac{\partial \vec{u}(\vec{r})}{\partial x_{i}}\right) \\
& +\frac{\partial}{\partial x_{i}}[\lambda(\vec{r}) \nabla \cdot \vec{u}(\vec{r})],
\end{aligned}
$$

where $\vec{u}$ is the displacement field. In general, $\vec{u}$ can be decoupled into a longitudinal part and a transverse part, i.e., $\vec{u}=\nabla \phi_{l}+\nabla \times\left(\phi_{t} \hat{e}_{z}\right)$, where $\phi_{l}$ and $\phi_{t}$ are the longitudinal and transverse gauge potentials, respectively. In the frequency domain, the solutions to $\phi_{l}$ and $\phi_{t}$ can be written as $\phi_{\alpha}(s)$ $=\Sigma_{m} a_{\alpha m}(s) J_{m}\left(k_{\alpha s} r\right) e^{i m \theta}(\alpha=l, t)$ in the cylinder and $\phi_{\alpha}(0)$

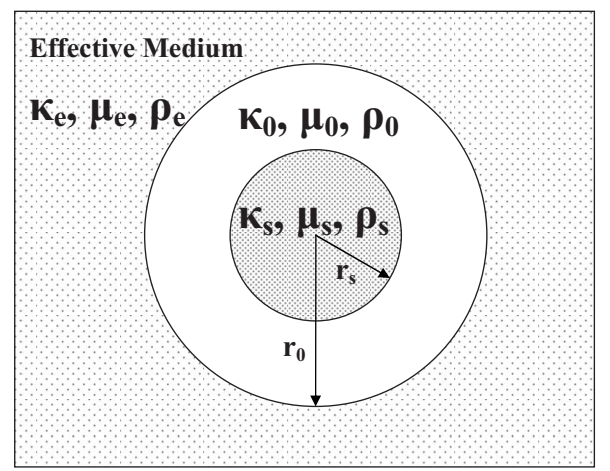

FIG. 1. Microstructure of the effective medium.

$=\sum_{m} a_{\alpha m}(0) J_{m}\left(k_{\alpha 0} r\right) e^{i m \theta}+b_{\alpha m}(0) H_{m}^{(1)}\left(k_{\alpha 0} r\right) e^{i m \theta}(\alpha=l, t)$ in the matrix. Here, $J_{m}(x)$ and $H_{m}^{(1)}(x)$ are, respectively, the Bessel and Hankel functions of the first kind, and $k_{l \delta}$ $=\omega \sqrt{\rho_{\delta} /\left(\lambda_{\delta}+2 \mu_{\delta}\right)}=\omega \sqrt{\rho_{\delta} /\left(\kappa_{\delta}+\mu_{\delta}\right)}$ and $k_{t \delta}=\omega \sqrt{\rho_{\delta} / \mu_{\delta}}$ are, respectively, the longitudinal and transverse wave vectors in the cylinder $(\delta=s)$ or in the matrix $(\delta=0)$, and $\omega$ is the angular frequency. The longitudinal and transverse waves in the matrix are coupled by the scatterings of the embedded cylinders. The coefficients of those Bessel and Hankel functions can be determined by considering the elastic boundary conditions, which are the continuities of the radial and tangential components of the displacement field, i.e., $u_{r}$ and $u_{\theta}$, and the continuities of the stresses, $\sigma_{r r}$ and $\sigma_{r \theta}$, at the interface. The stresses are defined as

$$
\sigma_{r r}=(\lambda+2 \mu) \frac{\partial u_{r}}{\partial r}+\lambda\left(\frac{1}{r} \frac{\partial u_{\theta}}{\partial \theta}+\frac{u_{r}}{r}\right)
$$

and

$$
\sigma_{r \theta}=\mu\left(\frac{1}{r} \frac{\partial u_{r}}{\partial \theta}+\frac{\partial u_{\theta}}{\partial r}-\frac{u_{\theta}}{r}\right) .
$$

These continuities on the surface of a cylinder relate $b_{l m}(0)$ and $b_{t m}(0)$ to $a_{l m}(0)$ and $a_{t m}(0)$ through $b_{l m}(0)$ $=D_{m}^{l l}(s, 0) a_{l m}(0)+D_{m}^{l t}(s, 0) a_{t m}(0)$ and $b_{t m}(0)=D_{m}^{t l}(s, 0) a_{l m}(0)$ $+D_{m}^{t t}(s, 0) a_{t m}(0)$, where $D_{m}^{\alpha \beta}(s, 0)(\alpha, \beta=l, t)$ are the Mie-like scattering coefficients determined by the boundary conditions at the interface between the cylinder and the matrix. $D_{m}^{\alpha \beta}(s, 0)(\alpha, \beta=l, t)$ are functions of $k_{l s}, k_{t s}, k_{l 0}, k_{t 0}$, and $r_{s .}{ }^{21}$ The explicit expressions for $D_{m}^{\alpha \beta}(s, 0)$ are displayed in Appendix A. The effective medium formulas for such a system can be derived by considering the scattering of elastic waves by a coated cylinder embedded in the effective medium with effective parameters $\left(\kappa_{e}, \mu_{e}, \rho_{e}\right) \cdot{ }^{6,9,22,23}$ Figure 1 shows the coated cylinder consisting of the cylindrical scatterer surrounded by a shell of the matrix. The inner and outer radii, which are denoted by $r_{s}$ and $r_{0}$, respectively, satisfy $\pi r_{s}^{2} / \pi r_{0}^{2}=p$, where $p$ is the filling fraction of the system. The effective parameters $\kappa_{e}, \mu_{e}$, and $\rho_{e}$ are determined by the condition that the total scattering of the coated cylinder vanishes. This condition together with the boundary conditions on the surface of the coated cylinder at $r=r_{0}$ provides another two relations: $b_{l m}(0)=D_{m}^{l l}(e, 0) a_{l m}(0)+D_{m}^{l t}(e, 0) a_{t m}(0)$ 
and $b_{t m}(0)=D_{m}^{t l}(e, 0) a_{l m}(0)+D_{m}^{t t}(e, 0) a_{t m}(0)$, where $D_{m}^{\alpha \beta}(e, 0)$ $(\alpha, \beta=l, t)$ can be obtained by replacing $\lambda_{s}, \rho_{s}, \mu_{s}, k_{l s}, k_{t s}$, and $r_{s}$ in $D_{m}^{\alpha \beta}(s, 0)(\alpha, \beta=l, t)$ with $\lambda_{e}, \rho_{e}, \mu_{e}, k_{l e}, k_{t e}$, and $r_{0}$, respectively. Here, $k_{l e}$ and $k_{t e}$ are the longitudinal and transverse wave vectors in the effective medium. These relations together with the previous relations give the following effective medium condition:

$$
D_{m}^{\alpha \beta}(e, 0)=D_{m}^{\alpha \beta}(s, 0) \quad(\alpha, \beta=l, t) .
$$

In the long wavelength limit where $k_{l 0} r_{0} \ll 1, k_{t 0} r_{0} \ll 1, k_{l e} r_{0}$ $\ll 1$, and $k_{t e} r_{0} \ll 1,{ }^{24}$ the following effective medium equations for the elastic metamaterial can be obtained:

$$
\begin{gathered}
\frac{\left(\kappa_{0}-\kappa_{e}\right)}{\left(\mu_{0}+\kappa_{e}\right)}=\frac{4 \widetilde{D}_{0}^{l l}(s, 0)}{i \pi r_{0}^{2} k_{l 0}^{2}}, \\
\frac{\left(\rho_{0}-\rho_{e}\right)}{\rho_{0}}=-\frac{8 \widetilde{D}_{1}^{l l}(s, 0)}{i \pi r_{0}^{2} k_{l 0}^{2}}, \\
\frac{\mu_{0}\left(\mu_{0}-\mu_{e}\right)}{\left[\kappa_{0} \mu_{0}+\left(\kappa_{0}+2 \mu_{0}\right) \mu_{e}\right]}=\frac{4 \widetilde{D}_{2}^{l l}(s, 0)}{i \pi r_{0}^{2} k_{l 0}^{2}},
\end{gathered}
$$

where $\widetilde{D}_{m}^{l l}(s, 0)$ is the $D_{m}^{l l}(s, 0)$ after taking the leading terms of $J_{m}(x)$ and $H_{m}^{(1)}(x)$ with $x \equiv k_{l 0} r_{s}$ and $k_{t 0} r_{s}$. The derivation of Eqs. (3a)-(3c) is given in Appendix B. It is interesting to point out that $\kappa_{e}, \rho_{e}$, and $\mu_{e}$ are independently determined by $\widetilde{D}_{m}^{l l}(s, 0)$ of the embedded cylinders alone, with $m=0,1$, and 2 , respectively. It should also be pointed out that Eqs. (3a)-(3c) require the wavelengths in both the host and the effective medium to be much larger than the size of the unit cell. However, it does not impose any restriction on the wavelengths inside the scatterer. If the condition of $k_{l s} r_{s} \ll 1$, $k_{t s} r_{s} \ll 1$ is further considered in $\widetilde{D}_{m}^{l l}(s, 0)$, the quasistatic limit is reached ${ }^{24}$ and Eqs. (3a)-(3c) become

$$
\begin{gathered}
\frac{\left(\kappa_{0}-\kappa_{e}\right)}{\left(\mu_{0}+\kappa_{e}\right)}=p \frac{\left(\kappa_{0}-\kappa_{s}\right)}{\left(\mu_{0}+\kappa_{s}\right)}, \\
\left(\rho_{0}-\rho_{e}\right)=p\left(\rho_{0}-\rho_{s}\right), \\
\frac{\left(\mu_{0}-\mu_{e}\right)}{\left[\kappa_{0} \mu_{0}+\left(\kappa_{0}+2 \mu_{0}\right) \mu_{e}\right]}=p \frac{\left(\mu_{0}-\mu_{s}\right)}{\left[\kappa_{0} \mu_{0}+\left(\kappa_{0}+2 \mu_{0}\right) \mu_{s}\right]},
\end{gathered}
$$

where $p$ is the filling fraction of the scatterer. The quasistatic limit requires that the wavelengths for both the longitudinal and transverse waves inside the scatterer are much larger than the size of the scatterer itself, which is not required in the long wavelength limit. In the limit of small $p$, Eqs. (4a) and (4c) converge to Eqs. (3.15) and (3.16) of Ref. 17. The three-dimensional results were reported by Berryman in Ref. 15 decades ago. It should be pointed out that the elastic effective medium theory cannot recover the acoustic effective medium theory by setting all the shear moduli to be zero, because the boundary conditions of elastic waves and acoustic waves are different.

\begin{tabular}{|c|c|c|c|c|}
\hline & $\begin{array}{c}\kappa_{e}+\mu_{e}>0 \\
\mu_{e}>0\end{array}$ & $\begin{array}{c}\kappa_{e}+\mu_{e}>0 \\
\mu_{e}<0\end{array}$ & $\begin{array}{c}\kappa_{e}+\mu_{e}<0 \\
\mu_{e}>0\end{array}$ & $\begin{array}{c}\kappa_{e}+\mu_{e}<0 \\
\mu_{e}<0\end{array}$ \\
\hline$\rho_{e}>0$ & $n_{l}>0 ; n_{t}>0$ & $n_{l}>0 ; t$ gap & $n_{t}>0 ; l$ : gap & $l, t$ : gap \\
\hline$\rho_{e}<0$ & $l, t:$ gap & $n_{t}<0 ; l:$ gap & $n_{l}<0 ; t$ : gap & $n_{l}<0 ; n_{t}<0$ \\
\hline
\end{tabular}

In nature, no elastic material has a negative mass density or a negative bulk or shear modulus. However, for metama-
TABLE I. Various wave propagation properties under different combinations of signs in $\rho_{e}, \mu_{e}$, and $\kappa_{e}+\mu_{e}$. Positive (negative) $n$ indicates positive (negative) propagating bands. $l$ and $t$ represent longitudinal and transverse waves, respectively.

terials, it is possible to achieve negative values in any of $\kappa_{e}$, $\rho_{e}$, and $\mu_{e}$ when the frequency is near the resonant frequencies of $\widetilde{D}_{m}^{l l}(s, 0), m=0,1$, and 2 . These negative effective parameters as well as their combinations can give rise to various interesting properties unseen in natural materials. For instance, since the effective phase velocities are $c_{l e}$ $=\sqrt{\lambda_{e}+2 \mu_{e}} \sqrt{1 / \rho_{e}}=\sqrt{\kappa_{e}+\mu_{e}} \sqrt{1 / \rho_{e}}$ and $c_{t e}=\sqrt{\mu_{e}} \sqrt{1 / \rho_{e}}$ for longitudinal and transverse waves, respectively, a single negative $\rho_{e}$ in a frequency window leads to imaginary $c_{l e}$ and $c_{t e}$, which, in turn, implies the existence of a simultaneous band gap for both longitudinal and transverse waves. On the other hand, simultaneous negative $\rho_{e}$ and $\mu_{e}$ make the refractive index negative for the transverse waves. Table I lists eight possible types of wave propagation in elastic metamaterials with different combinations of signs in $\kappa_{e}+\mu_{e}, \rho_{e}$, and $\mu_{e}$. Here, it should be pointed out that the effective bulk modulus and shear modulus $\kappa_{e}$ and $\mu_{e}$ determined according to Eq. (3) do not satisfy the well-known bounds ${ }^{25,26}$ on the effective elastic moduli as these bounds are derived in the quasistatic limit. This has been pointed out previously (see Ref. 9) in the study of the effective medium properties of acoustic metamaterials.

\section{APPLICATION TO SIMPLE ELASTIC METAMATERIALS}

In order to search for elastic metamaterials with different types of wave propagation, we start from the following simple metamaterial: a triangular lattice of silicone rubber cylinders with radius $0.3 a$ ( $a$ is the lattice constant) embedded in an epoxy host. ${ }^{27}$ Since silicone rubber possesses very small longitudinal and transverse wave speeds, wavelengths inside the silicone rubber cylinder may be comparable to or even much smaller than the size of the cylinder at low frequencies. Thus, Mie-like resonances may occur, which serve as the built-in resonances required for metamaterials. Figure 2(a) shows the band structures near the $\Gamma$ point calculated by the multiple-scattering method ${ }^{21,28}$ in dimensionless frequency $\tilde{f}=(\omega / 2 \pi)\left(a / c_{t 0}\right)$, where $c_{t 0}=\sqrt{\mu_{0}} \sqrt{1 / \rho_{0}}$. Multigap structures associated with various resonances are clearly seen in the frequency range $\tilde{f} \leqslant 0.05$. In this system, the long wavelength conditions hold when $\tilde{f} \ll 0.3$. In Figs. 2(b), 2(c), and 2(d), the real parts of $\kappa_{e}, \rho_{e}$, and $\mu_{e}$ calculated from Eq. (3) are plotted, respectively. The imaginary parts are 15 orders of magnitude smaller and can be ignored. It is clear that Figs. 2(b)-2(d) exhibit very rich resonant behaviors for each 


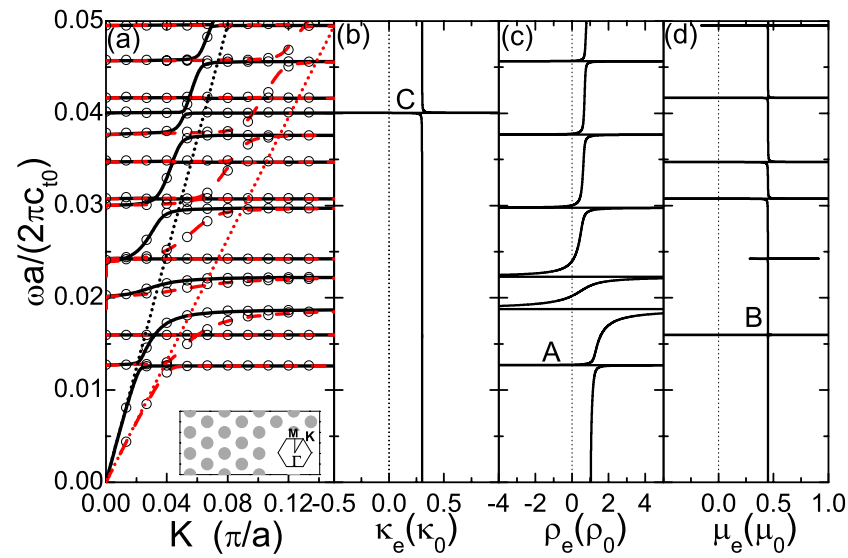

FIG. 2. (Color online) (a) Dispersion relation along the $\Gamma K$ direction for a triangular lattice of silicone rubber cylinders $\left(r_{s}\right.$ $=0.3 a$ ) embedded in epoxy host. Open circles are obtained by bandstructure calculation, curves are determined from Eqs. (3a)-(3c), and solid black and dashed red curves represent longitudinal and transverse branches, respectively. Dotted lines are obtained from Eqs. (4a)-(4c). (b), (c), and (d) are the effective bulk modulus, effective mass density, and effective shear modulus obtained from Eqs. (3a)-(3c), respectively.

of the three effective medium parameters even at such low frequencies. The dispersion curves of the system can also be obtained from Eq. (3) through $\omega=\left(\sqrt{\kappa_{e}+\mu_{e}} / \sqrt{\rho_{e}}\right) K$ (longitudinal) and $\omega=\left(\sqrt{\mu_{e}} / \sqrt{\rho_{e}}\right) K$ (transverse). These results are also plotted in Fig. 2(a) in solid and dashed curves for the longitudinal and transverse branches, respectively. The excellent agreement between the open circles and solid (or dashed) curves shows the validity and accuracy of Eq. (3). The dotted lines in Fig. 2(a) denote the dispersion curves obtained from the quasistatic theory, i.e., Eq. (4), which gives the following frequency independent effective parameters: $\rho_{e}=1.0333 \rho_{0}$, $\lambda_{e}=0.24824 \lambda_{0}$, and $\mu_{e}=0.44935 \mu_{0}$. Obviously, the quasistatic theory fails to predict the band structures above the lowest resonance. In Fig. 2, a few narrow band gaps for both longitudinal and transverse waves can be found in the regions of single negative $\rho_{e}$. Two obvious ones are at $\tilde{f}$ $=(0.01871,0.02011)$ and $(0.02222,0.02391)$. Figure 2 also exhibits regions of single negative $\kappa_{e}$ or $\mu_{e}$. However, no double negative region is found in Fig. 2. Even such a simple system can already produce various types of wave propagation listed in Table I. We also notice that the regions possessing negative effective parameters shown in Fig. 2 are too narrow to be useful. In the next section, we will demonstrate how to use Eq. (3) as a guide to engineer the scatterers to search for more interesting properties.

\section{APPLICATION TO COMPLICATED ELASTIC METAMATERIALS}

In the following two examples, we will show how to engineer the scatterers $\left[\right.$ or $\left.\widetilde{D}_{m}^{l l}(s, 0)\right]$ in a way to (i) widen the gaps shown in Fig. 2(a), which is equivalent to enlarging the negative regions of $\rho_{e}$, and (ii) create a band with negative
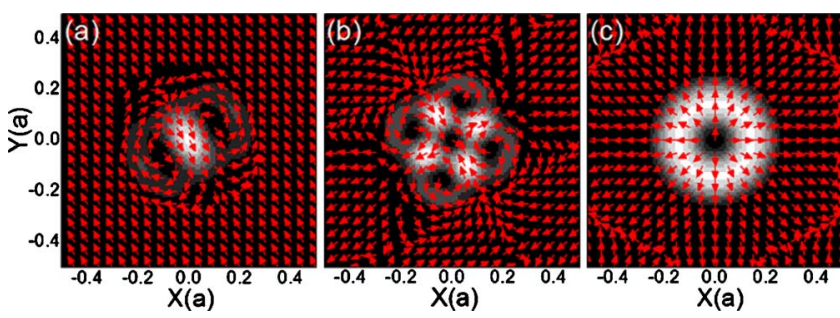

FIG. 3. (Color online) (a), (b), and (c) correspond to the displacement fields at frequencies marked by "A," "B," and "C" in Fig. 1, respectively. Brightness represents the magnitude and arrows denote the directions.

refractive index, which is equivalent to making the negative regions of both $\rho_{e}$ and $\mu_{e}$ or/and $\kappa_{e}+\mu_{e}$ overlap. For these purposes, we need to understand the mode structures near the resonances of $\rho_{e}, \mu_{e}$, and $\kappa_{e}$. In Figs. 3(a), 3(b), and 3(c), we plot the displacement fields of the Bloch states at the $\Gamma$ point corresponding to the lowest resonances of $\rho_{e}, \mu_{e}$, and $\kappa_{e}$, respectively, as marked by "A," "B," and "C" in Fig. 2. The brightness and the arrows indicate, respectively, the magnitude and the direction of the displacement field $\vec{u}$ at a certain time. Figure 3(a) shows that the core part of the silicone rubber scatterer oscillates as a whole. This mode can be regarded as a simple "mass-spring" harmonic oscillator, with the core part serving as "mass" and the edge part serving as "spring." Figure 3(b) exhibits that the shape of the silicone rubber cylinder is deformed in oscillation, with its crosssectional area conserved, and Fig. 3(c) shows that the shape of the silicone rubber cylinder is fixed, with its crosssectional area oscillating in time. The above mode structures provide useful guidance for the engineering of the silicone rubber cylinders. For instance, replacing the inner region of silicone rubber with another heavier cylinder, e.g., lead, ${ }^{27}$ will enhance the field oscillation of the silicone rubber, which, in turn, will widen the resonant region of $\rho_{e}$. It is straightforward to generalize the effective medium theory of Eq. (3) to the case where the scatterers are layered cylinders. In this case, the quantities $\widetilde{D}_{m}^{l l}(s, 0)$ can be obtained by using the standard transfer-matrix method. ${ }^{21}$ Figure 4(a) shows the results of both band-structure calculations (in open circles) and Eq. (3) (in solid and dashed curves) when the radius of the lead cylinder is $r_{\text {lead }}=0.9 r_{s}$. Compared to Fig. 2(a), it is interesting to see that the lead replacement has significantly widened the negative region of $\rho_{e}$ at the first resonance as shown in Fig. 4(b) and a large band gap with gap-midgap ratio $\Delta \tilde{f} / \tilde{f}_{c}=0.74$ is created. Thus, Eq. (3) can be used to search for metamaterials which possess large $\Delta \tilde{f} / \tilde{f}_{c}$. Figure 4(c) shows the calculated $\Delta \widetilde{f} / \tilde{f}_{c}$ as a function of $r_{\text {lead }} / r_{s}$ at different values of $r_{s} / a$. It is seen that a ratio as large as $\Delta \tilde{f} / \tilde{f}_{c}=1.2$ can be achieved. It should be mentioned that the above three-component metamaterial has been studied before. ${ }^{11}$ Recently, the analysis of a simple model based on a stiff matrix also suggests a negative effective mass density in such materials. ${ }^{13}$ However, the model cannot provide any information on the effective moduli.

In the following, we use Eq. (3) to search for elastic metamaterials with negative refractive index. In this case, the 


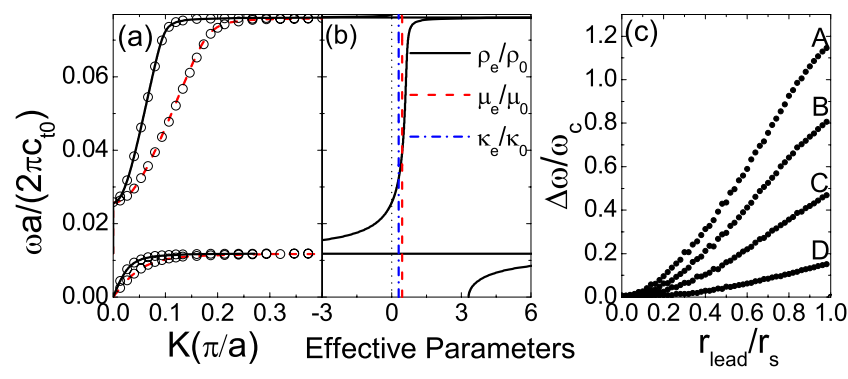

FIG. 4. (Color online) (a) The same as in Fig. 1(a), but the central region of the silicone rubber cylinder is replaced with a lead cylinder with radius $r_{l e a d}=0.9 r_{s}$. (b) The effective parameters, i.e., $\rho_{e}$ (solid and black line), $\mu_{e}$ (dashed and red line), and $\kappa_{e}$ (dashdotted and blue line), of the above system obtained from Eqs. (3a)-(3c). (c) Gap-midgap ratio obtained from Eqs. (3a)-(3c). A, B, $\mathrm{C}$, and $\mathrm{D}$ correspond to $r_{s}=0.4 a, 0.3 a, 0.2 a$, and $0.1 a$, respectively.

resonances of both $\rho_{e}$ and $\mu_{e}$ or/and $\kappa_{e}$ need to be considered. Figures 3(b) and 3(c) suggest that if we want to enhance these resonances, we need to make the scatterer easier to deform and compress. Thus, we replace the core region of the silicone rubber with air. ${ }^{27}$ The radius of the air core $r_{\text {air }}$ is chosen such that the negative regions of both $\rho_{e}$ and $\mu_{e}$ or/ and $\kappa_{e}$ overlap. The results of band-structure calculation with $r_{\text {air }}=0.87 r_{s}$ are shown in Fig. 5(a) in open circles, which coincide with the results of Eq. (3) shown in solid and dashed curves. In the region of $0.12240<\tilde{f}<0.12253$, negative- $n$ bands of both longitudinal and transverse waves are found, which implies that $\rho_{e}, \kappa_{e}+\mu_{e}$, and $\mu_{e}$ are all negative. These negative values are induced only by the resonances of $\rho_{e}$ and $\mu_{e}$ as shown in Fig. 5(b). While in another region of $0.12340<\tilde{f}<0.12356$, a negative- $n$ band is found only for longitudinal waves, which implies that $\rho_{e}$ and $\kappa_{e}$ $+\mu_{e}$ are both negative. These negative values arise from resonances of both $\rho_{e}$ and $\kappa_{e}$. Figure 5(a) shows that there exist small discrepancies between the band-structure calculation and the effective medium prediction for the longitudinal branches. The reason is that the values of $k_{l 0} r_{0}$ and $k_{t 0} r_{0}$ are not much less than 1 for the frequencies shown in Fig. 5(a).

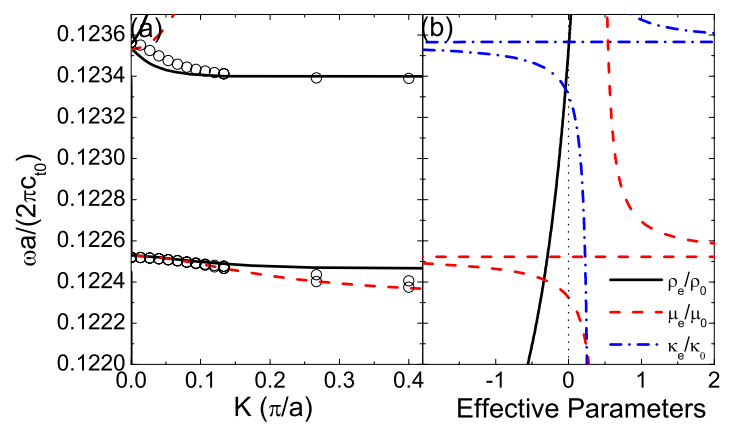

FIG. 5. (Color online) (a) The same as in Fig. 1(a), but the central region of the silicone rubber cylinder is replaced with an air cylinder with radius $r_{a i r}=0.87 r_{s}$. (b) The effective parameters, i.e., $\rho_{e}$ (solid and black line), $\mu_{e}$ (dashed and red line), and $\kappa_{e}$ (dashdotted and blue line), of the above system obtained from Eqs. (3a)-(3c).
Thus, the approximation which was used to derive Eq. (3) becomes less accurate. For example, when $\tilde{f}=0.1230, k_{l 0} r_{0}$ $=0.185$, and $k_{t 0} r_{0}=0.406$, the errors induced by taking leading terms are $5 \%$ in $H_{0}^{(1)}\left(k_{l 0} r_{0}\right), 0.1 \%$ in $H_{1}^{(1)}\left(k_{l 0} r_{0}\right), 0.02 \%$ in $H_{2}^{(1)}\left(k_{l 0} r_{0}\right), 3 \%$ in $H_{0}^{(1)}\left(k_{t 0} r_{0}\right), 0.9 \%$ in $H_{1}^{(1)}\left(k_{t 0} r_{0}\right)$, and $0.2 \%$ in $H_{2}^{(1)}\left(k_{t 0} r_{0}\right)$. Thus, the errors introduced are larger in $H_{0}^{(1)}$ than in $H_{1}^{(1)}$ and $H_{2}^{(1)}$. In other words, $\kappa_{e}$ is less accurate than $\rho_{e}$ and $\mu_{e}$. Therefore, the discrepancies between bandstructure calculation and effective medium prediction can only be found for the longitudinal branches, which involves $\kappa_{e}$, but not for transverse branches.

\section{CONCLUSIONS}

In conclusion, we have developed an effective medium theory for certain two-dimensional elastic metamaterials. Such materials are composed of isotropic scatterers embedded in a solid host in a triangular structure. The theory was derived in the long wavelength limit, where the wavelengths in both the host and the effective medium are large compared to the size of the unit cell, while the wavelengths in the scatterer can be comparable to or smaller than the size of the scatterer itself. The theory shows that the effective parameters, $\kappa_{e}, \rho_{e}$, and $\mu_{e}$, are independently determined and can turn negative near resonances. Comparing this theory with the band-structure calculations of three different elastic metamaterials shows explicitly that the theory is accurate in the long wavelength limit, even near resonances, where the quasistatic theory fails. The theory not only yields a better understanding of various types of wave propagation in elastic metamaterials under a unified framework, but also provides a convenient tool to search for metamaterials with desired properties. We have demonstrated two examples of designing elastic metamaterials: one with a large band gap at low frequencies, and the other with double negative bands for both longitudinal and transverse branches.

Finally, it is worth noting that the dispersion relations for elastic waves are, in general, not isotropic near the $\Gamma$ point, even at low frequencies. ${ }^{29}$ However, it can be shown analytically that isotropy holds for the case of the triangular lattice considered here.

\section{ACKNOWLEDGMENT}

The work was supported by Hong Kong RGC Grant No. 604703.

\section{APPENDIX A: CALCULATING THE SCATTERING COEFFICIENTS $D_{m}^{\alpha \beta}(s, 0)$}

The coefficients $D_{m}^{\alpha \beta}(s, 0)$, determined by boundary conditions, connect the scattered field to the incident field at each scatterer. The explicit expressions of $D_{m}^{\alpha \beta}(s, 0)$ take the following forms:

$$
D_{m}^{l l}(s, 0)=\frac{1}{M_{m}}\left|\begin{array}{llll}
a_{1}^{l} & b_{1}^{t} & c_{1}^{l} & c_{1}^{t} \\
a_{2}^{l} & b_{2}^{t} & c_{2}^{l} & c_{2}^{t} \\
a_{3}^{l} & b_{3}^{t} & c_{3}^{l} & c_{3}^{t} \\
a_{4}^{l} & b_{4}^{t} & c_{4}^{l} & c_{4}^{t}
\end{array}\right|,
$$




$$
\begin{aligned}
& D_{m}^{l t}(s, 0)=\frac{1}{M_{m}}\left|\begin{array}{llll}
a_{1}^{t} & b_{1}^{t} & c_{1}^{l} & c_{1}^{t} \\
a_{2}^{t} & b_{2}^{t} & c_{2}^{l} & c_{2}^{t} \\
a_{3}^{t} & b_{3}^{t} & c_{3}^{l} & c_{3}^{t} \\
a_{4}^{t} & b_{4}^{t} & c_{4}^{l} & c_{4}^{t}
\end{array}\right|, \\
& D_{m}^{t l}(s, 0)=\frac{1}{M_{m}}\left|\begin{array}{llll}
b_{1}^{l} & a_{1}^{l} & c_{1}^{l} & c_{1}^{t} \\
b_{2}^{l} & a_{2}^{l} & c_{2}^{l} & c_{2}^{t} \\
b_{3}^{l} & a_{3}^{l} & c_{3}^{l} & c_{3}^{t} \\
b_{4}^{l} & a_{4}^{l} & c_{4}^{l} & c_{4}^{t}
\end{array}\right|, \\
& D_{m}^{t t}(s, 0)=\frac{1}{M_{m}}\left|\begin{array}{llll}
b_{1}^{l} & a_{1}^{t} & c_{1}^{l} & c_{1}^{t} \\
b_{2}^{l} & a_{2}^{t} & c_{2}^{l} & c_{2}^{t} \\
b_{3}^{l} & a_{3}^{t} & c_{3}^{l} & c_{3}^{t} \\
b_{4}^{l} & a_{4}^{t} & c_{4}^{l} & c_{4}^{t}
\end{array}\right|,
\end{aligned}
$$

(A1c)

where

$$
M_{m}=\left|\begin{array}{llll}
b_{1}^{l} & b_{1}^{t} & c_{1}^{l} & c_{1}^{t} \\
b_{2}^{l} & b_{2}^{t} & c_{2}^{l} & c_{2}^{t} \\
b_{3}^{l} & b_{3}^{t} & c_{3}^{l} & c_{3}^{t} \\
b_{4}^{l} & b_{4}^{t} & c_{4}^{l} & c_{4}^{t}
\end{array}\right| .
$$

The matrix elements in Eqs. (A1a)-(A1d) have the following forms:

$$
\begin{gathered}
a_{1}^{l}=-k_{l 0} J_{m}^{\prime}\left(k_{l 0} r_{s}\right) \\
a_{2}^{l}=\frac{i m}{r_{s}} J_{m}\left(k_{l 0} r_{s}\right) \\
a_{3}^{l}=-\left(\lambda_{0}+2 \mu_{0}\right) k_{l 0}^{2} J_{m}^{\prime \prime}\left(k_{l 0} r_{s}\right)-\frac{\lambda_{0}}{r_{s}} k_{l 0} J_{m}^{\prime}\left(k_{l 0} r_{s}\right) \\
+m^{2} \frac{\lambda_{0}}{r_{s}^{2}} J_{m}\left(k_{l 0} r_{s}\right), \\
a_{4}^{l}=-2 \frac{i m}{r_{s}} \mu_{0} k_{l 0} J_{m}^{\prime}\left(k_{l 0} r_{s}\right)+2 \frac{i m}{r_{s}^{2}} \mu_{0} J_{m}\left(k_{l 0} r_{s}\right) \\
a_{4}^{t}=\mu_{0} k_{t 0}^{2} J_{m}^{\prime \prime}\left(k_{t 0} r_{s}\right)-\frac{\mu_{0}}{r_{s}} k_{t 0} J_{m}^{\prime}\left(k_{t 0} r_{s}\right)+\mu_{0} \frac{m^{2}}{r_{s}^{2}} J_{m}\left(k_{t 0} r_{s}\right) \\
a_{1}^{t}=-\frac{i m}{r_{s}} J_{m}\left(k_{t 0} r_{s}\right) \\
a_{2}^{t}=-k_{t s} J_{m}^{\prime}\left(k_{t 0} r_{s}\right) \\
a_{3}^{t}=2 \frac{i m}{r_{s}^{2}} \mu_{0} J_{m}\left(k_{t 0} r_{s}\right)-2 \frac{i m}{r_{s}} \mu_{0} k_{t 0} J_{m}^{\prime}\left(k_{t 0} r_{s}\right)
\end{gathered}
$$

$$
\begin{aligned}
b_{3}^{l}= & \left(\lambda_{0}+2 \mu_{0}\right) k_{l 0}^{2} H_{m}^{(1)^{\prime \prime}}\left(k_{l 0} r_{s}\right)+\frac{\lambda_{0}}{r_{s}} k_{l 0} H_{m}^{(1)^{\prime}}\left(k_{l 0} r_{s}\right) \\
& -m^{2} \frac{\lambda_{0}}{r_{s}^{2}} H_{m}^{(1)}\left(k_{l 0} r_{s}\right), \\
b_{4}^{l}= & 2 \frac{i m}{r_{s}} \mu_{0} k_{l 0} H_{m}^{(1)^{\prime}}\left(k_{l 0} r_{s}\right)-2 \frac{i m}{r_{s}^{2}} \mu_{0} H_{m}^{(1)}\left(k_{l 0} r_{s}\right),
\end{aligned}
$$$$
b_{1}^{t}=\frac{i m}{r_{s}} H_{m}^{(1)}\left(k_{t 0} r_{s}\right),
$$$$
b_{2}^{t}=k_{t 0} H_{m}^{(1)^{\prime}}\left(k_{t 0} r_{s}\right) \text {, }
$$

$$
b_{3}^{t}=-2 \frac{i m}{r_{s}^{2}} \mu_{0} H_{m}^{(1)}\left(k_{t 0} r_{s}\right)+2 \frac{i m}{r_{s}} \mu_{0} k_{t 0} H_{m}^{(1)^{\prime}}\left(k_{t 0} r_{s}\right),
$$

$$
b_{4}^{t}=-\mu_{0} k_{t 0}^{2} H_{m}^{(1)^{\prime \prime}}\left(k_{t 0} r_{s}\right)+\frac{\mu_{0}}{r_{s}} k_{t 0} H_{m}^{(1)^{\prime}}\left(k_{t 0} r_{s}\right)-\mu_{0} \frac{m^{2}}{r_{s}^{2}} H_{m}^{(1)}\left(k_{t 0} r_{s}\right)
$$

$$
\begin{gathered}
c_{1}^{l}=-k_{l s} J_{m}^{\prime}\left(k_{l s} r_{s}\right), \\
c_{2}^{l}=\frac{i m}{r_{s}} J_{m}\left(k_{l s} r_{s}\right), \\
c_{3}^{l}=-\left(\lambda_{s}+2 \mu_{s}\right) k_{l s}^{2} J_{m}^{\prime \prime}\left(k_{l s} r_{s}\right)-\frac{\lambda_{s}}{r_{s}} k_{l s} J_{m}^{\prime}\left(k_{l s} r_{s}\right)+m^{2} \frac{\lambda_{s}}{r_{s}^{2}} J_{m}\left(k_{l s} r_{s}\right),
\end{gathered}
$$

$$
c_{4}^{l}=-2 \frac{i m}{r_{s}} \mu_{s} k_{l s} J_{m}^{\prime}\left(k_{l s} r_{s}\right)+2 \frac{i m}{r_{s}^{2}} \mu_{s} J_{m}\left(k_{l s} r_{s}\right)
$$

$$
c_{1}^{t}=-\frac{i m}{r_{s}} J_{m}\left(k_{t s} r_{s}\right)
$$

$$
c_{2}^{t}=-k_{t s} J_{m}^{\prime}\left(k_{t s} r_{s}\right) \text {, }
$$

$$
c_{3}^{t}=2 \frac{i m}{r_{s}^{2}} \mu_{s} J_{m}\left(k_{t s} r_{s}\right)-2 \frac{i m}{r_{s}} \mu_{s} k_{t s} J_{m}^{\prime}\left(k_{t s} r_{s}\right),
$$

$$
c_{4}^{t}=\mu_{s} k_{t s}^{2} J_{m}^{\prime \prime}\left(k_{t s} r_{s}\right)-\frac{\mu_{s}}{r_{s}} k_{t s} J_{m}^{\prime}\left(k_{t s} r_{s}\right)+\mu_{s} \frac{m^{2}}{r_{s}^{2}} J_{m}\left(k_{t s} r_{s}\right) .
$$




\section{APPENDIX B: DERIVATION OF EFFECTIVE MEDIUM EQUATIONS}

Taking $J_{0}(x) \cong 1-\left(x^{2} / 4\right)$ and $H_{0}^{(1)}(x) \cong i(2 / \pi) \ln x$, for $x$ $\equiv k_{l e} r_{0}, k_{t e} r_{0}, k_{l 0} r_{0}$, and $k_{t 0} r_{0}$ in $D_{m}^{\alpha \beta}(e, 0)$ when $m=0$, we find, to the lowest order in $\omega$,

$$
\begin{aligned}
D_{0}^{l l}(e, 0) \cong \frac{i \pi r_{0}^{2} \omega^{2} \rho_{0}\left(\lambda_{0}+\mu_{0}-\lambda_{e}-\mu_{e}\right)}{4\left(\lambda_{0}+2 \mu_{0}\right)\left(\mu_{0}+\lambda_{e}+\mu_{e}\right)} & =\frac{i \pi r_{0}^{2} k_{l 0}^{2}\left(\kappa_{0}-\kappa_{e}\right)}{4\left(\kappa_{e}+\mu_{0}\right)}, \\
D_{0}^{l t}(e, 0), D_{0}^{t l}(e, 0), D_{0}^{t t}(e, 0) & \cong 0 .
\end{aligned}
$$

We take the same approximations in $J_{0}(x)$ and $H_{0}^{(1)}(x)$ as above for $x \equiv k_{l 0} r_{s}$ and $k_{t 0} r_{s}$ in $D_{0}^{\alpha \beta}(s, 0)$ to obtain

$$
\begin{gathered}
D_{0}^{l l}(s, 0) \cong \widetilde{D}_{0}^{l l}(s, 0), \\
D_{0}^{l t}(s, 0), D_{0}^{t l}(s, 0), D_{0}^{t t}(s, 0) \cong 0 .
\end{gathered}
$$

Equations (B1a) and (B1b) together with Eqs. (B2a) and (B2b) and the effective medium condition, i.e., Eq. (2), gives Eq. (3a).

Also, when $m=1$, the Bessel and Hankel functions in $D_{1}^{\alpha \beta}(e, 0)$ take the forms of $J_{1}(x) \cong(x / 2)-\left(x^{3} / 16\right)$ and $H_{1}^{(1)}(x) \cong i[(x / \pi) \ln (x / 2)-(2 / \pi x)]$, for $x \equiv k_{l e} r_{0}, k_{t e} r_{0}, k_{l 0} r_{0}$, and $k_{t 0} r_{0}$. Then $D_{1}^{\alpha \beta}(e, 0)$ changes to

$$
\begin{gathered}
D_{1}^{l l}(e, 0) \cong-\frac{i \pi r_{0}^{2} \omega^{2}\left(\rho_{0}-\rho_{e}\right)}{8\left(\lambda_{0}+2 \mu_{0}\right)}=-\frac{i \pi r_{0}^{2} k_{l 0}^{2}\left(\rho_{0}-\rho_{e}\right)}{8 \rho_{0}}, \\
D_{1}^{l t}(e, 0)=-D_{1}^{t l}(e, 0) \cong \frac{k_{t 0}}{k_{l 0}} \frac{\pi r_{0}^{2} \omega^{2}\left(\rho_{0}-\rho_{e}\right)}{8\left(\lambda_{0}+2 \mu_{0}\right)}, \\
D_{1}^{t t}(e, 0) \cong-\left(\frac{k_{t 0}}{k_{l 0}}\right)^{2} \frac{i \pi r_{0}^{2} \omega^{2}\left(\rho_{0}-\rho_{e}\right)}{8\left(\lambda_{0}+2 \mu_{0}\right)} .
\end{gathered}
$$

The same approximation of $J_{1}(x)$ and $H_{1}^{(0)}(x)$ can be taken for $x \equiv k_{l 0} r_{s}$ and $k_{t 0} r_{s}$ in $D_{1}^{\alpha \beta}(s, 0)$. Then $D_{1}^{\alpha \beta}(s, 0)$ changes into the following forms:

$$
\begin{gathered}
D_{1}^{l l}(s, 0) \cong \widetilde{D}_{1}^{l l}(s, 0), \\
D_{1}^{l t}(s, 0)=-D_{1}^{t l}(s, 0) \cong i \frac{k_{t 0}}{k_{l 0}} \widetilde{D}_{1}^{l l}(s, 0),
\end{gathered}
$$

$$
D_{1}^{t t}(s, 0) \cong\left(\frac{k_{t 0}}{k_{l 0}}\right)^{2} \widetilde{D}_{1}^{l l}(s, 0) .
$$

It is clearly seen that $D_{1}^{l t}(e, 0), D_{1}^{t l}(e, 0)$, and $D_{1}^{t t}(e, 0)$ are proportional to $D_{1}^{l l}(e, 0)$ in the same ways as $D_{1}^{l t}(s, 0)$, $D_{1}^{t l}(s, 0)$, and $D_{1}^{t t}(s, 0)$ are proportional to $D_{1}^{l l}(s, 0)$. Thus, Eq. (2) gives four identical equations, which have the form shown in Eq. (3b).

Similarly, when $m=2$, we have $J_{2}(x) \cong\left(x^{2} / 8\right)-\left(x^{4} / 96\right)$, $H_{2}^{(1)}(x) \cong i\left[-\left(4 / \pi x^{2}\right)-(1 / \pi)\right]$ for $x \equiv k_{l e} r_{0}, k_{t e} r_{0}, k_{l 0} r_{0}$, and $k_{t 0} r_{0}$ in $D_{2}^{\alpha \beta}(e, 0)$ and obtain

$$
\begin{gathered}
D_{2}^{l l}(e, 0) \cong \frac{i \pi r_{0}^{2} \omega^{2} \mu_{0} \rho_{0}\left(\mu_{0}-\mu_{e}\right)}{4\left(\lambda_{0}+2 \mu_{0}\right)\left[\kappa_{0} \mu_{0}+\left(\kappa_{0}+2 \mu_{0}\right) \mu_{e}\right]} \\
=\frac{i \pi r_{0}^{2} k_{l 0}^{2} \mu_{0}\left(\mu_{0}-\mu_{e}\right)}{4\left[\kappa_{0} \mu_{0}+\left(\kappa_{0}+2 \mu_{0}\right) \mu_{e}\right]}, \\
D_{2}^{l t}(e, 0)=-D_{2}^{t l}(e, 0) \\
\cong-\left(\frac{k_{t 0}}{k_{l 0}}\right)^{2} \frac{\pi r_{0}^{2} \omega^{2} \mu_{0} \rho_{0}\left(\mu_{0}-\mu_{e}\right)}{4\left(\lambda_{0}+2 \mu_{0}\right)\left[\kappa_{0} \mu_{0}+\left(\kappa_{0}+2 \mu_{0}\right) \mu_{e}\right]}, \\
D_{2}^{t t}(e, 0) \cong\left(\frac{k_{t 0}}{k_{l 0}}\right)^{4} \frac{i \pi r_{0}^{2} \omega^{2} \mu_{0} \rho_{0}\left(\mu_{0}-\mu_{e}\right)}{4\left(\lambda_{0}+2 \mu_{0}\right)\left[\kappa_{0} \mu_{0}+\left(\kappa_{0}+2 \mu_{0}\right) \mu_{e}\right]} .
\end{gathered}
$$

Again, after taking the same approximation of $J_{2}(x)$ and $H_{2}^{(1)}(x)$ for $x \equiv k_{l 0} r_{s}$ and $k_{t 0} r_{s}$ in $D_{2}^{\alpha \beta}(s, 0)$, we get the following expressions for $D_{2}^{\alpha \beta}(s, 0)$ :

$$
\begin{gathered}
D_{2}^{l l}(s, 0) \cong \widetilde{D}_{2}^{l l}(s, 0) \\
D_{2}^{l t}(s, 0)=-D_{2}^{t l}(s, 0) \cong i\left(\frac{k_{t 0}}{k_{l 0}}\right)^{2} \widetilde{D}_{2}^{l l}(s, 0), \\
D_{2}^{t t}(s, 0) \cong\left(\frac{k_{t 0}}{k_{l 0}}\right)^{4} \widetilde{D}_{2}^{l l}(s, 0) .
\end{gathered}
$$

We find again that $D_{2}^{l t}(e, 0), D_{2}^{t l}(e, 0)$, and $D_{2}^{t t}(e, 0)$ are proportional to $D_{2}^{l l}(e, 0)$, with the same ratios as those appearing in the ratios of $D_{2}^{l t}(s, 0), D_{2}^{t l}(s, 0)$, and $D_{2}^{t t}(s, 0)$ to $D_{2}^{l l}(s, 0)$, respectively. Then, the last effective medium equation, i.e., Eq. (3c), can be obtained.

\footnotetext{
*Author to whom correspondence should be addressed; phzzhang@ust.hk

${ }^{1}$ D. R. Smith, W. J. Padilla, D. C. Vier, S. C. Nemat-Nasser, and S. Schultz, Phys. Rev. Lett. 84, 4184 (2000); R. A. Shelby, D. R. Smith, S. C. Nemat-Nasser, and S. Schultz, Appl. Phys. Lett. 78, 489 (2001); R. A. Shelby, D. R. Smith, and S. Schultz, Science 292, 77 (2001).
}

2 J. B. Pendry, Phys. Rev. Lett. 85, 3966 (2000); N. Garcia and M. Nieto-Vesperinas, ibid. 88, 207403 (2002); J. T. Shen and P. M. Platzman, Appl. Phys. Lett. 80, 3286 (2002); D. R. Smith, D. Schurig, M. Rosenbluth, S. Schultz, S. A. Ramarkrishna, and J. B. Pendry, ibid. 82, 1506 (2003); P. Kolinko and D. R. Smith, Opt. Express 11, 640 (2003).

${ }^{3}$ D. R. Smith and J. B. Pendry, J. Opt. Soc. Am. B 23, 391 (2006); 
D. R. Smith, J. B. Pendry, and M. Wiltshire, Science 305, 788 (2004); T. Koschny, M. Kafesaki, E. N. Economou, and C. M. Soukoulis, Phys. Rev. Lett. 93, 107402 (2004); N. Fang, D. Xi, J. Xu, M. Ambati, W. Srituravanich, C. Sun, and X. Zhang, Nat. Mater. 5, 452 (2006).

${ }^{4}$ J. B. Pendry, A. J. Holden, D. J. Robbins, and W. J. Stewart, IEEE Trans. Microwave Theory Tech. 47, 2075 (1999); J. B. Pendry, A. J. Holden, W. J. Stewart, and I. Youngs, Phys. Rev. Lett. 76, 4773 (1996).

${ }^{5}$ L. Lewin, Proc. Inst. Electr. Eng. 94, 65 (1947); A. N. Lagarkov, A. K. Sarychev, Y. R. Smychkovich, and A. P. Vinogradov, J. Electromagn. Waves Appl. 6, 1159 (1992); A. K. Sarychev, R. C. McPhedran, and V. M. Shalaev, Phys. Rev. B 62, 8531 (2000).

${ }^{6}$ Y. Wu, J. Li, Z. Q. Zhang, and C. T. Chan, Phys. Rev. B 74, 085111 (2006).

${ }^{7}$ Stephen O'Brien and J. B. Pendry, J. Phys.: Condens. Matter 14, 4035 (2002).

${ }^{8}$ Th. Koschny, P. Markoš, E. N. Economou, D. R. Smith, D. C. Vier, and C. M. Soukoulis, Phys. Rev. B 71, 245105 (2005).

${ }^{9}$ J. Li and C. T. Chan, Phys. Rev. E 70, 055602(R) (2004).

${ }^{10}$ J. Mei, Z. Liu, W. Wen, and P. Sheng, Phys. Rev. Lett. 96, 024301 (2006).

${ }^{11}$ Z. Liu, X. Zhang, Y. Mao, Y. Y. Zhu, Z. Yang, C. T. Chan, and P. Sheng, Science 289, 1734 (2000).

${ }^{12}$ C. Goffaux, J. Sánchez-Dehesa, A. L. Yeyati, Ph. Lambin, A. Khelif, J. O. Vasseur, and B. Djafari-Rouhani, Phys. Rev. Lett. 88, 225502 (2002).

${ }^{13}$ Z. Liu, C. T. Chan, and P. Sheng, Phys. Rev. B 71, 014103 (2005).

${ }^{14}$ A. A. Krokhin, J. Arriaga, and L. N. Gumen, Phys. Rev. Lett. 91, 264302 (2003).
${ }^{15}$ J. G. Berryman, J. Acoust. Soc. Am. 68, 1809 (1980).

${ }^{16}$ S. Torquato, Phys. Rev. Lett. 79, 681 (1997).

${ }^{17}$ Y. Kantor and D. J. Bergman, J. Mech. Phys. Solids 30, 355 (1982).

${ }^{18}$ Israel Cohen and David J. Bergman, Phys. Rev. B 68, 024104 (2003).

${ }^{19}$ M. S. Kushwaha, P. Halevi, G. Martínez, L. Dobrzynski, and B. Djafari-Rouhani, Phys. Rev. B 49, 2313 (1994).

${ }^{20}$ J. O. Vasseur, P. A. Deymier, B. Chenni, B. Djafari-Rouhani, L. Dobrzynski, and D. Prevost, Phys. Rev. Lett. 86, 3012 (2001).

${ }^{21}$ Y. Lai, Ph.D. thesis, Hong Kong University of Science and Technology, 2005.

${ }^{22}$ M. Kafesaki, R. S. Penciu, and E. N. Economou, Phys. Rev. Lett. 84, 6050 (2000).

${ }^{23} \mathrm{P}$. Sheng, Introduction to Wave Scattering, Localization, and Mesoscopic Phenomena (Academic, San Diego, 1995).

${ }^{24}$ W. Lamb, D. M. Wood, and N. W. Ashcroft, Phys. Rev. B 21, 2248 (1980).

${ }^{25}$ Z. Hashin and S. Shtrikman, J. Mech. Phys. Solids 11, 127 (1963).

${ }^{26}$ S. Torquato, Appl. Mech. Rev. 44, 37 (1991).

${ }^{27}$ The silicone rubber considered has $\rho=1.3 \times 10^{3} \mathrm{~kg} / \mathrm{m}^{3}, \lambda=6$ $\times 10^{5} \mathrm{~N} / \mathrm{m}^{2}$, and $\mu=4 \times 10^{4} \mathrm{~N} / \mathrm{m}^{2}$. The corresponding parameters in the epoxy host are $\rho=1.18 \times 10^{3} \mathrm{~kg} / \mathrm{m}^{3}, \lambda=4.43$ $\times 10^{9} \mathrm{~N} / \mathrm{m}^{2}$, and $\mu=1.59 \times 10^{9} \mathrm{~N} / \mathrm{m}^{2}$. For lead, $\rho=11.6$ $\times 10^{3} \mathrm{~kg} / \mathrm{m}^{3}, \lambda=4.23 \times 10^{10} \mathrm{~N} / \mathrm{m}^{2}$, and $\mu=1.49 \times 10^{10} \mathrm{~N} / \mathrm{m}^{2}$. For air, $\rho=1.23 \mathrm{~kg} / \mathrm{m}^{3}$ and $k=\lambda=1.42 \times 10^{10} \mathrm{~N} / \mathrm{m}^{2}$. The above parameters are obtained from Ref. 11.

${ }^{28}$ M. Kafesaki and E. N. Economou, Phys. Rev. B 60, 11993 (1999); J. Mei, Z. Liu, J. Shi, and D. Tian, ibid. 67, 245107 (2003).

${ }^{29}$ Q. Ni and J. Cheng, Phys. Rev. B 72, 014305 (2005). 